\title{
Causas del desprendimiento de retina y el desenlace visual final en menores de 18 años en el Hospital San Vicente Fundación
}

\author{
Claudia Andrea Echeverry-Aguilar' ${ }^{1}$ Martha Lía Gaviria-Bravo²
}

\section{RESUMEN}

Objetivo: caracterizar las causas del desprendimiento de retina y determinar la agudeza visual final en menores de 18 años en el Hospital San Vicente Fundación entre 2012 y 2017.

Metodología: se realizó un análisis retrospectivo de historias clínicas de pacientes con diagnóstico de desprendimiento de retina confirmado por examen oftalmológico o ecografía, se incluyeron pacientes menores de 18 años que ingresaron al Hospital San Vicente Fundación entre 2012 y 2017 para identificar las causas del desprendimiento de retina y la agudeza visual final.

Resultados: se analizaron 51 historias clínicas: 28 hombres y 23 mujeres, 39,1 \% ocurrieron en menores de 1 año y no se encontró desprendimiento de retina por encima de los 14 años. Las causas se establecieron como retinopatía de la prematuridad $23,5 \%$, trauma ocular 21,6\%, retinoblastoma 9,8\%, toxoplasmosis congénita 7,8\%, toxocara 7,8\%, entre otras, no se encontró causa en $3,9 \%$ de los pacientes.

En 50 ojos de 41 pacientes se determinó la agudeza visual final, de los cuales 47 (94\%) quedaron con agudeza visual peor o igual a 20/200 y 3 ojos con agudeza visual de 20/40 o mejor.

Discusión: el desprendimiento de retina es infrecuente en los niños, sin embargo, sus causas y desenlaces son más devastadores que en los adultos. En nuestro medio las principales causas son la retinopatía de la prematuridad, el trauma, el retinoblastoma y las infecciones parasitarias. Diferente a los adultos, en los niños se requiere una evaluación más cuidadosa y un umbral de sospecha más bajo para considerar enfermedades potencialmente mortales.

1 Residente de Oftalmología, Universidad de Antioquia, Medellín, Colombia.

2 Oftalmología Pediátrica y Estrabismo, Hospital San Vicente Fundación, Medellín, Colombia.

Correspondencia: Claudia Andrea Echeverry Aguilar; echeverryclau@gmail.com

Recibido: agosto 6 de 2018

Aceptado: octubre 17 de 2018

Cómo citar: Echeverry-Aguilar CA, Gaviria-Bravo ML. Causas del desprendimiento de retina y el desenlace visual final en menores de 18 años en el Hospital San Vicente Fundación. latreia. 2019 0ct-Dic;32(4):259-265. D0I 10.17533/udea.iatreia.29. 


\section{PALABRAS CLAVE}

Contusiones; Desprendimiento de Retina; Heridas Penetrantes; Retina; Retinoblastoma; Retinopatía de la Prematuridad

\section{SUMMARY}

Causes of retinal detachment and visual outcome in children under 18 years old treated at Hospital Universitario de San Vicente Fundación

Objective: To characterize the causes of retinal detachment and to determinate the visual outcome in children younger than 18 years old evaluated at San Vicente Fundación Hospital between 2012 and 2017.

Methodology: We performed a retrospective analysis of medical records of patients with a diagnosis of retinal detachment confirmed by ophthalmological examination or ultrasound scan. Patients under 18 years old admitted to San Vicente Fundación Hospital between 2012 and 2017 were included to identify the causes of retinal detachment and the final visual acuity.

Results: 51 clinical histories were analyzed: 28 men and 23 women; $39.1 \%$ occurred in children under 1 year old, and no retinal detachment was found above 14 years old. The causes were established as: retinopathy of prematurity $23.5 \%$, ocular trauma $21.6 \%$, retinoblastoma $9.8 \%$, congenital toxoplasmosis $7.8 \%$, toxocara $7.8 \%$, among others. The cause was not found in $3.9 \%$ of patients.

In 50 eyes of 41 patients, the final visual acuity was determined, of which 47 (94\%) had visual acuity equal or worse to $20 / 200$ and 3 eyes with better corrected visual acuity of $20 / 40$ or better.

Discussion: Retinal detachment is infrequent in children; however, its causes and outcomes are more devastating than in adults. In our environment, the main causes are retinopathy of prematurity, trauma, retinoblastoma and parasitic infections; unlike adults, children require a more careful assessment and a lower threshold of suspicion to consider life-threatening diseases.

\section{KEY WORDS}

Contusions, Retina; Retinal Detachment; Retinoblastoma; Retinopathy of Prematurity; Wounds, Penetrating

\section{INTRODUCCIÓN}

El desprendimiento de retina (DR) es la separación entre el neuroepitelio y el epitelio piomentario de la retina que, por diferentes mecanismos, llevará a la disminución de la agudeza visual, visión de fotopsias, miodesopsias $y$, dependiendo de la causa subyacente $y$ de la instauración de un manejo temprano o tardío, producirá secuelas visuales permanentes en diferente medida ${ }^{(1-3)}$. Su incidencia en la población general es baja, entre 0,03-0,01\%, y de esta estadística el 3,2-6,6 \% es aportado por los menores de 18 años ${ }^{(1-3)}$.

En los niños esta condición tiende a ser más dramática por varias razones: dificultades para expresar sus síntomas, dependencia de sus cuidadores para recibir atención oftalmológica, manifestaciones menos específicas como leucocoria, tropiezos o dificultad de los niños para desenvolverse en ambientes desconocidos, llevándolos a recibir una atención tardía. Además, en los niños es mayor el riesgo de ambliopía y vitreorretinopatía proliferativa muy agresiva que obstaculiza más su recuperación ${ }^{(4,5)}$.

Al ser el DR en niños una entidad de baja incidencia, en la literatura se encuentran pocas series de casos publicadas al respecto y de estas la mayoría se refiere a un tipo de DR específico, con pocos estudios que analicen las causas de todos los DR. Recientemente, fue publicado en Alemania el resultado de las características clínicas y el desenlace de una cohorte de 87 pacientes menores de 18 años con DR, en los cuales se encontró como principal causa el trauma ocular (40\%), seguido de anomalías oculares congénitas o del desarrollo (37\%), cirugía ocular previa (27 \%) y miopía (23\%), pero se excluyeron los pacientes con retinopatía de la prematuridad ${ }^{(5)}$.

Actualmente, se desconocen las características de esta condición en los niños de nuestro medio; sin embargo, cada vez más se encuentran diferencias significativas entre los DR de los adultos y los niños en otros entornos, particularmente en cuanto a sus causas y desenlaces, lo que hace necesaria su evaluación por separado para mejorar su comprensión y enfoque ${ }^{(1,2,5)}$. El objetivo de este estudio fue determinar las causas del DR en menores de 18 años en el Hospital San Vicente Fundación desde junio de 2012 hasta febrero de 2017, describir sus principales características $y$ el resultado visual final. 


\section{METODOLOGÍA}

Se realizó un estudio descriptivo y retrospectivo de historias clínicas de pacientes con diagnósticos de DR en menores de 18 años que ingresaron al Hospital San Vicente Fundación de la ciudad de Medellín entre junio de 2012 y febrero de 2017. Se identificaron variables demográficas (edad y género), bilateralidad, causas deI desprendimiento de retina, tipo de DR y la agudeza visual final.

Se encontraron 55 pacientes con diagnóstico de DR, sus historias clínicas fueron revisadas por un investigador quien determinó si cumplían con los criterios de inclusión: diagnóstico de DR confirmado por examen oftalmológico o ecográfico y ser menor de 18 años. Se excluyeron cuatro pacientes: dos que presentaron lesiones predisponentes para el DR sin desprendimiento de retina $y$ dos que informaron antecedentes de DR que no se logró documentar en la historia clínica. Los pacientes incluidos fueron ingresados a una base de datos donde se registró la edad al momento del diagnóstico, sexo, causa del DR, tipo de DR y la agudeza visual final. Esta última se documentó solo en 50 ojos correspondientes a 41 pacientes, el resto de los pacientes abandonaron el proceso de seguimiento.

La información se ingresó y analizó en el paquete estadístico SPSS, versión 19. Las variables cualitativas se describen según su número absoluto y relativo. Las variables continuas se describen con medianas y rango intercuartil (RIC) El estudio contó con el aval del Comité de investigación del Hospital San Vicente Fundación.

\section{RESULTADOS}

Se incluyeron 51 pacientes menores de 18 años con diagnóstico de DR confirmado, 28 hombres (54,9\%) y 23 mujeres, para un total de 64 ojos con desprendimiento de retina, de los cuales 13 pacientes tenían compromiso bilateral. La mediana en la edad de la población fue de 3 años (RIC: 4 meses - 7 años), la edad mínima fue de 1 mes y la máxima 13 años, la mayoría de los DR ocurrieron en menores de 1 año $(39,1 \%)$ (Tabla 1).
Tabla 1. Variables demográficas de pacientes con DR $<18$ años, Hospital San Vicente Fundación 2012-2017

\begin{tabular}{ccccc}
\hline Variables & $\begin{array}{c}\text { Pacientes } \\
\text { (n) }\end{array}$ & $\begin{array}{c}\text { Pacientes } \\
\text { (\%) }\end{array}$ & $\begin{array}{c}\text { Ojos } \\
\text { (n) }\end{array}$ & $\begin{array}{c}\text { Ojos } \\
(\%)\end{array}$ \\
\hline Hombre & 28 & 54 & 38 & 59,4 \\
\hline Mujeres & 23 & 45 & 26 & 40,6 \\
\hline 0 & \multicolumn{4}{c}{ Edad (años) } \\
\hline 1 & 16 & 30,8 & 25 & 39,1 \\
\hline 2 & 1 & 1,9 & 1 & 1,6 \\
\hline 3 & 3 & 5,8 & 3 & 4,7 \\
\hline 4 & 5 & 9,6 & 6 & 9,4 \\
\hline 5 & 4 & 7,7 & 4 & 6,3 \\
\hline 6 & 4 & 7,7 & 4 & 6,3 \\
\hline 7 & 3 & 5,8 & 3 & 4,7 \\
\hline 8 & 2 & 3,8 & 3 & 4,7 \\
\hline 9 & 1 & 1,9 & 1 & 1,6 \\
\hline 10 & 5 & 9,6 & 5 & 7,8 \\
\hline 11 & 0 & 0 & 0 & 0 \\
\hline 12 & 2 & 3,8 & 3 & 4,7 \\
\hline 13 & 4 & 7,7 & 4 & 6,3 \\
\hline $14-18$ & 2 & 3,8 & 2 & 3,1 \\
\hline & 0 & 0 & 0 & 0 \\
\hline & & & & \\
\hline
\end{tabular}

Fuente: elaboración propia.

Se logró conocer la causa deI DR de 49 pacientes. Las principales causas se establecieron como retinopatía de la prematuridad (ROP) en 12 pacientes (18 ojos), trauma ocular en 11 pacientes ( 8 traumas abiertos y 3 traumas cerrados), manifestación inicial de retinoblastoma en 5 pacientes ( 5 ojos), toxoplasmosis congénita en 4 pacientes (6 ojos) y toxocariasis en 4 pacientes (4 ojos). En 2 pacientes (2 ojos) no se encontró la causa del DR (Tabla 2).

Los pacientes con DR secundario a ROP provenían de otras ciudades: Turbo, Valledupar, Bucaramanga y de otras instituciones de Medellín, ninguno había nacido ni había recibido sus cuidados neonatales en el Hospital San Vicente Fundación.

Según los registros clínicos, los DR se clasificaron como traccional, regmatógeno (DR producido como consecuencia de un agujero o desgarro en la retina), seroso (DR por acumulación de líquido debajo de la retina) o desconocido. 20 ojos tenían DR traccional (31,3\%), 12 ojos DR regmatógeno (18,8 \%), 13 ojos DR seroso (20,3 \%) y 19 ojos DR de tipo desconocido (29,7 \%). 
Tabla 2. Causas de DR en pacientes < 18 años, Hospital San Vicente Fundación 2012-2017

\begin{tabular}{|c|c|c|c|c|}
\hline Causas & Pacientes (n) & Pacientes (\%) & Ojos (n) & Ojos (\%) \\
\hline Retinopatía de la prematuridad & 12 & 23,5 & 18 & 28,1 \\
\hline Trauma Ocular & 11 & & 11 & \\
\hline Abierto & 8 & 21,6 & 8 & 17,2 \\
\hline Cerrado & 3 & & 3 & \\
\hline Retinoblastoma & 5 & 9,8 & 5 & 7,8 \\
\hline Toxoplasmosis congénita & 4 & 7,8 & 6 & 9,4 \\
\hline Toxocara & 4 & 7,8 & 4 & 6,3 \\
\hline Miopía degenerativa & 3 & 5,9 & 5 & 7,8 \\
\hline Persistencia de la vasculatura fetal & 3 & 5,9 & 4 & 6,3 \\
\hline Enfermedad de Coats & 2 & 3,9 & 2 & 3,1 \\
\hline Síndrome de Vogt Koyanagi Harada (VKH) & 1 & 2 & 2 & 3,1 \\
\hline Degeneraciones periféricas de la retina & 1 & 2 & 1 & 1,6 \\
\hline Síndrome de Marfan & 1 & 2 & 1 & 1,6 \\
\hline Síndrome de Stickler & 1 & 2 & 1 & 1,6 \\
\hline Vitreorretinopatía hereditaria & 1 & 2 & 2 & 3,1 \\
\hline Desconocida & 2 & 3,9 & 2 & 3,1 \\
\hline Total & 51 & 100 & 64 & 100 \\
\hline
\end{tabular}

Fuente: elaboración propia.

En 50 ojos correspondientes a 41 pacientes se logró determinar la agudeza visual final, 5 ojos requirieron enucleación por diagnóstico final de retinoblastoma del grupo E (estadio más grave de retinoblastoma intraocular), 21 ojos no tenían percepción luminosa, 11 ojos con percepción de luz, 2 ojos percibían movimientos de manos, 3 ojos lograban contar dedos, el resto de ojos se registraron en notación Snellen: 1 ojo 20/800, 1 ojo 20/400, 3 ojos 20/200, 1 ojo 20/40, 1 ojo 20/30 y 1 ojo 20/25, para un total de 47 ojos (94\%) con agudeza visual final igual o peor a 20/200, y 3 ojos (6 \%) con agudeza visual mejor corregida de 20/40 o mejor ${ }^{(6)}$.

\section{DISCUSIÓN}

EI DR es raro en niños, sus causas son diferentes a las de los adultos y sus desenlaces anatómicos y visuales más devastadores ${ }^{(1-3)}$. Nuestro estudio evaluó de manera retrospectiva y por un periodo de 5 años las características de los niños menores de 18 años con DR. Se incluyeron 51 pacientes, de los cuales la proporción de hombres $(54,9 \%$ ) y mujeres ( $45 \%$ ) fue similar, con un ligero predominio del sexo masculino. En otros estudios se reporta un predominio masculino, probablemente porque la principal causa del desprendimiento de retina encontrada por ellos fue el trauma ${ }^{(1,2,5,7-9)}$. La causa principal del desprendimiento de retina en nuestros pacientes fue la retinopatía de Ia prematuridad (23,5\%), seguida por el trauma (21,6 $\%)$. Estos hallazoos no coinciden con lo reportado en la literatura que indica como principales causas las anomalías en el desarrollo y el trauma, esta diferencia se debe a que nosotros incluimos la retinopatía de la prematuridad $y$ en los otros estudios esta entidad no ha sido tenida en cuenta ${ }^{(5)}$.

En nuestro estudio la mayor proporción de estos traumas fueron abiertos, mientras que en la literatura mundial se reporta con más frecuencia el trauma cerrado ${ }^{(1,4,5)}$. La tercera causa en nuestro grupo fue la manifestación inicial de retinoblastoma (9,8 \%), no se encontraron otros artículos que reportaran esta patología como una causa importante de DR en niños.

El retinoblastoma usualmente se manifiesta por otros signos como leucocoria o estrabismo y al fondo del ojo suele encontrarse una masa blanca, vascularizada; sin embargo, los retinoblastomas de tipo exofítico o difusos pueden manifestarse por desprendimiento de retina, lo cual dificulta y retrasa el diagnóstico ${ }^{(6,10,11)}$. Los 
cinco pacientes de nuestro estudio con retinoblastoma como causa del DR ya tenían una enfermedad avanzada al momento del diagnóstico (tumor grupo E) y todos requirieron enucleación. Por lo anterior, un desprendimiento de retina unilateral en un niño lactante, prescolar o escolar debe alertar y hacer sospechar la presencia de retinoblastoma ${ }^{(6)}$. La cuarta causa fue la toxoplasmosis congénita, que cada vez es menos reportada en la literatura por medidas adoptadas durante la gestación para prevenir la infección y realizar un manejo que permita limitar la enfermedad congénita. Es probable que en el futuro esta disminución también se vea reflejada en las estadísticas de nuestro país ${ }^{(4)}$.

La mayor proporción de ojos afectados (25\%) se presentó en niños menores de 1 año de edad y su principal causa fue DR traccional secundario a retinopatía de la prematuridad, seguido por los pacientes prescolares y escolares en quienes predominaron los traumas oculares, manifestación inicial de retinoblastoma e infecciones parasitarias.

La menor incidencia se presentó en los pacientes preadolescentes y adolescentes donde las causas más importantes fueron la miopía degenerativa y las enfermedades hereditarias. Encontramos mayor incidencia de DR en niños menores con una mediana de edad de 3 años, a diferencia de otros estudios donde la mediana se ha encontrado en 10 a 12 años, como ya hemos mencionado esta diferencia se debe a que los otros estudios excluyeron la retinopatía de la prematuridad ${ }^{(1,8,9,12)}$.

En nuestro estudio el tipo de DR encontrado en mayor proporción fue traccional $(31,3 \%)$ a diferencia de lo que se encuentra en los adultos donde el tipo más frecuente es el regmatógeno, esto se correlaciona con las diferencias en la etiología de cada grupo etario ${ }^{(3)}$.

Se determinó la agudeza visual final en 50 ojos de 41 pacientes. Cinco ojos (10\%), que correspondían a ojos con retinoblastoma avanzado, fueron enucleados $y$ 42 ojos quedaron con agudeza visual igual o peor a 20/200, resultando en un mal desenlace para la mayoría de los ojos (94\%). El mal pronóstico de la mayoría de los ojos está acorde con el pobre desenlace conocido en los niños con desprendimiento de retina debido a vitreorretinopatía proliferativa, diagnóstico tardío, etiologías relacionadas con el desarrollo y enfermedades congénitas ${ }^{(1,4,5)}$. Solo 3 ojos terminaron con agudeza visual corregida de 20/40 o mejor, de éstos, 2 ojos tenían síndrome de VKH y un ojo trauma cerrado (Tabla 3 ).

\section{Tabla 3. Agudeza visual final}

\begin{tabular}{|cccc|}
\hline Agudeza visual final & & Ojos (n) & Ojos (\%) \\
\hline Enucleados & & 5 & 10 \\
\hline No percepción luminosa & & 21 & 42 \\
\hline Percepción luminosa & & 11 & 22 \\
\hline Movimiento de manos & & 2 & 4 \\
\hline Cuenta dedos & & 3 & 6 \\
\hline 20/800 (snellen) & $1,6 \log M A R$ & 1 & 2 \\
\hline $20 / 400$ (snellen) & $1,3 \log M A R$ & 1 & 2 \\
\hline $20 / 200$ (snellen) & $1 \log$ MAR & 3 & 6 \\
\hline $20 / 40$ (snellen) & $0,3 \log M A R$ & 1 & 2 \\
\hline $20 / 30$ (snellen) & $0,18 \log$ MAR & 1 & 2 \\
\hline $20 / 25$ (snellen) & $0,1 \log$ MAR & 1 & 2 \\
\hline Total & & 50 & 100 \\
\hline
\end{tabular}

Fuente: elaboración propia.

En los últimos años se ha avanzado mucho en los programas de tamización, prevención y manejo de la retinopatía de la prematuridad, por lo que con una adecuada implementación de estos programas el riesgo de desarrollar desprendimiento de retina es mínimo. Una vez se ha producido el desprendimiento de retina en la ROP, sin embargo, aún con cirugía los resultados visuales son pobres ${ }^{(13-15)}$.

Por tratarse de un estudio retrospectivo no fue posible estandarizar un mayor número de variables que nos permitieran obtener más información. Se sugiere para futuras investigaciones realizar análisis prospectivos donde se incluyan todas las causas del desprendimiento de retina, las características de su manejo y el desenlace en los niños. No disponemos de otros estudios locales con un objetivo similar que nos permita comparar los resultados. En nuestro estudio el retinoblastoma fue la tercera causa del desprendimiento de retina con el peor desenlace. Es necesario que el médico general y el médico rural estén entrenados para realizar el examen de fondo de ojo en los niños, ya que, por nuestro sistema de salud, son ellos los primeros a quienes tienen acceso los pacientes. Deben estar capacitados para sospechar y hacer un diagnóstico temprano de retinoblastoma. Si el retinoblastoma es 
detectado en un estadio inicial, con los tratamientos que tenemos disponibles en la actualidad es posible conservar la vida, el globo ocular y, en muchos casos, la visión ${ }^{(6,16)}$.

Como conclusión, en nuestro medio, las causas del desprendimiento de retina en niños son diferentes a las reportadas en la literatura mundial, por lo que es fundamental ser conscientes de esto para sospecharlas y tratarlas. Infortunadamente, también encontramos que la gran mayoría de ojos con DR quedaron ciegos o con baja visión, aunque las principales causas del DR eran prevenibles. Este panorama se puede modificar mejorando los cuidados neonatales de los prematuros, principalmente el manejo del oxígeno, implementando medidas que permitan prevenir el trauma ocular en niños, entrenando a los profesionales de la salud para lograr un diagnóstico temprano de retinoblastoma, ampliando la cobertura de agua potable para prevenir la infección por quistes del toxoplasma y desparasitando las mascotas ${ }^{(3,4,6,6,17)}$.

\section{CONFLICTOS DE INTERESES}

Ninguno por declarar.

\section{AGRADECIMIENTOS}

Se agradece al servicio de registros hospitalarios del Hospital San Vicente Fundación por proporcionar la información de los pacientes con diagnóstico de desprendimiento de retina $y$ al Dr. Oscar Villada, epidemiólogo de la Dirección de Investiogaciones del Hospital San Vicente Fundación por su asesoría. A la Dra. María Elena González, oftalmóloga oncóloga por su continuo trabajo de sensibilización sobre retinoblastoma.

\section{REFERENCIAS BIBLIOGRÁFICAS}

1. Meier P. Netzhautablösung im Kindesalter: Differenzialdiagnose und aktuelle Therapieoptionen. Klin MonbI Augenheilkd. 2008;225(09):779-90. DOI 10.1055/s-2008-1027515.

2. Rumelt S, Sarrazin L, Averbukh E, Halpert M, Hemo I. Paediatric $\nabla$ s adult retinal detachment. Eye (Lond). 2007;21:1473-8. DOI 10.1038/sj.eye.6702511.
3. Cano Reyes JC, Infante Tavio NI, González Guerrero L, Fernández Pérez SR, Herrera Cutié D. Desprendimiento de retina: una revisión bibliográfica necesaria. Medisan. 2015;19(1):78-87.

4. Snead MP Retinal detachment in childhood. En: Lambert S, Lyons C. Taylor and Hoyt's Pediatric Ophthalmology and Strabismus. 5a nd. Edinburgh: Elsevier; 2016. p. 546-61.

5. Barth T, Zeman F, Helbig H, Oberacher-Velten I. Clinical Features and Outcome of Paediatric Retinal Detachment. Ophthalmologica. 2017;237(2):63-72. DOI 10.1159/000455355.

6. Gallie BL, Soliman SE. Retinoblastoma. En: Lambert S, Lyons C. Taylor and Hoyt's Pediatric Ophthalmology and Strabismus. 5a nd. Edinburgh: Elsevier; 2016. p. 424-42.

7. Fong A, Yip P, Kwok T, CW T. A 12-year review on the aetiology and surgical outcomes of paediatric rhegmatogenous retinal detachments in Hong Kong. Eye (Lond). 2015;30(3):355-61. DOI 10.1038/eye.2015.212.

8. Gurler B, Coskun E, Öner V, Gómez A, Erbagci I. Clinical characteristics and surgical outcomes of pediatric rhegmatogenous retinal detachment. Int Ophthalmol. 2016;36(4):521-5. DOI 10.1007/s10792-015-0158-3.

9. Chen SN, Lian IeB, Wei YJ. Epidemiology and clinical characteristics of rhegmatogenous retinal detachment in Taiwan. Br J Ophthalmol. 2016;100(9):121620. DOI 10.1136/bjophthalmol-2015-307481.

10. Shields CL, Ghassemi F, Tuncer S, Thangappan A, Shields JA. Clinical Spectrum of Diffuse Infiltrating Retinoblastoma in 34 Consecutive Eyes. Ophthalmology. 2008;115(12):2253-8. DOI 10.1016/j. ophtha.2008.07.003.

11. Shields CL, Schoenberg E, Kocher K, Shukla SY, Kaliki S, Shields JA. Lesions simulating retinoblastoma (Pseudoretinoblastoma) in 604 cases: Results based on age at presentation. Ophthalmology. 2013;120(2):3116. DOI 10.1016/j.ophtha.2012.07.067.

12. Poulsen CD, Peto T, Grauslund J, Green A. Epidemiologic characteristics of retinal detachment surgery at a specialized unit in Denmark. Acta Ophtalmol. 2016;94(6):548-55. DOI 10.1111/aos.13113.

13. Fierson WM, American Academy of Pediatrics Section on Ophthalmology, American Academy of Ophthalmology, American Association for Pediatric Ophthalmology and Strabismus, American Association of 
Certified Orthoptists. Screening Examination of Premature Infants for Retinopathy of Prematurity. Pediatrics. 2013;131(1):189-95. DOI 10.1542/peds.2012-2996.

14. OPS. Guía De Práctica Clínica Para El Manejo De La Retinopatía De La Prematuridad. Washington, D.C: OMS; 2018.

15. Yang MB. Retinopathy of Prematurity. In: Lambert SR, Lyons C, editors. Taylor and Hoyt's Pediatric Ophthalmology and Strabismus. 5a nd. Edinburohh: Elsevier; 2016. p. 299-305.
16. Rodriguez-Galindo C, Wilson MW, Dyer M. Retinoblastoma. In: Orkin S, Nathan D, Ginsburg D, Look T, Fisher D, Lux S, editors. Nathan and Oski's Hematology and Oncology of Infancy and Childhood. 8a nd. London: Elsevier; 2015. p. 1747-1778.

17. Oréfice F, Santos $\nabla$, Daniel $\nabla$, Cynthia AC, Lambert Oréfice J, Alves Costa R. Toxoplasmosis. In: Foster S, Vitale A, editors. Diagnosis and treatment of Uveitis. 2 nd. London: Jaype-Highlights Medical Publishers; 2013. p. 543-69. 